\title{
RESERCH ON SPATIAL AND TEMPORAL DISTRIBUTION OF COLOR STEEL BUILDING BASED ON MULTI-SOURCE HIGH-RESOLUTION SATELLITE IMAGERY
}

\author{
S. W. Yang ${ }^{1,2,}$, J. J. Ma ${ }^{1,2}$, J. M. Wang ${ }^{1,2}$ \\ (1. Lanahou jiaotong University, Faculty of Geomatics, Lanzhou 730070; \\ 2. Gansu Provincial Engineering Laboratory for National Geographic State Monitoring, Lanzhou \\ 730070)
}

\begin{abstract}
KEY WORDS: Color Steel Building, High-resolution Satellite Imagery, Spatial and Temporal Distribution, Urban Space Form, Coupling Relationship
\end{abstract}

\begin{abstract}
:
As representative vulnerable regions of the city, dense distribution areas of temporary color steel building are a major target for control of fire risks, illegal buildings, environmental supervision, urbanization quality and enhancement for city's image. In the domestic and foreign literature, the related research mainly focuses on fire risks and violation monitoring. However, due to temporary color steel building's special characteristics, the corresponding research about temporal and spatial distribution, and influence on urban spatial form etc. has not been reported. Therefore, firstly, the paper research aim plans to extract information of large-scale color steel building from high-resolution images. Secondly, the color steel plate buildings were classified, and the spatial and temporal distribution and aggregation characteristics of small (temporary buildings) and large (factory building, warehouse, etc.) buildings were studied respectively. Thirdly, the coupling relationship between the spatial distribution of color steel plate and the spatial pattern of urban space was analysed. The results show that there is a good coupling relationship between the color steel plate building and the urban spatial form. Different types of color steel plate building represent the pattern of regional differentiation of urban space and the phased pattern of urban development.
\end{abstract}

\section{INTRODUCTION}

The color steel buildings are constructed with color steel plates as main materials, and are widely used in temporary residential houses, construction sheds, warehouses, factories and kiosks. In recent years, because of the urbanization, urban transformation and upgrading and real estate development, many temporary color steel buildings have been produced in urban sites, such as urban villages, urban-rural junctions, urban fringes, and new economic development zones. These buildings are an important product of the urbanization. At the same time, the new urban fragile areas are also formed in the densely-distributed areas which become the focus of environmental supervision, urbanization quality and improvement of urban image.

Urban space form is the carrier of urban spatial structure (Woo et al., 2011) . In recent years, scholars have studied the urban space patterns from the different perspectives and have achieved a large number of research results (Zhong et al., 2014; Li, 2015). Urban space form represents the geographic location, spatial distribution characteristics and the combination of the urban functional areas. Their changes directly or indirectly reflect the problems of the urban environmental changes, population changes and the socio-economic development (Feng et al., 2011). Among them, the buildings create the space shape of the city, reflecting the spatial characteristics of the urban development the degree of development and the internal imbalance of the urban and other issues (Zhang et al., 2013). The color steel building is one of the urban buildings. Its spatial distribution and agglomeration characteristics are closely related to the internal economic development of the urban, population structure differences, location and function.

At present through literature analysis, we have not found the related research on the evolution relationship between the color steel building and the urban space form. However, there were some studies of the urban problems using night-time lighting, green areas and roads (Shi et al., 2014). For example, the commercial development, high-density residential and lowdensity residential can effectively represent the landscape and spatial structure of the city (Herold et al., 2002). Some studies extracted and analysed the dynamics and characteristics of the urban expansion based on DMSP/OLS, SPOT-VGT data (Liu et al., 2012; Pandey et al., 2013). Other studies analysed the impact and evolution of the urban space form and urban agglomerations by using high-speed railways (Shen et al., 2014; Yin et al., 2015; Chen, 2018).

High-resolution satellite remote sensing images can effectively display the detailed information of the ground features. Therefore, the paper extracted the color steel building information based on domestic high-resolution No. 2 image (GF-2) and analysed the spatial distribution and gathering characteristics of the color steel buildings in Anning District, Lanzhou City by GIS spatial analysis methods. The purpose of the research is to reveal the evolution of the current urban spatial structure and the influencing factors from another perspective.

\section{DATA AND METHODS}

\subsection{Study Area}

Lanzhou City, Gansu Province is a typical western valley city. Anning District is located on the north bank of the Yellow River. The district is built for later, but it has developed rapidly in recent years. The process of urbanization has been remarkable. The large enterprises in the region are clustered and have the only national-level economic and technological development zone in Gansu Province. The tertiary institutions in the district are concentrated and there are many large-scale parks. Therefore, the proportion of greenery is higher. At

\footnotetext{
* Corresponding author: Yang Shuwen - ysw040966@163.com; 825198827@qq.com
} 
present, Anning district is in the period of urban transformation and upgrading. Temporary color steel buildings in urban villages, urban-rural junctions, urban fringes and new technology development areas are widely distributed and agglomeration characteristics are obvious.

\subsection{Color Steel Building Extraction based on High- Resolution Images}

The color steel buildings have the characteristics of the scattered space distribution, different sizes of patch area, types and high and low differences, etc. High-resolution satellite remote sensing images have the high spatial resolution and the abundant spatial information. Therefore, it is an effective mean for extracting the color steel buildings in a large area.

The domestic high-resolution No. 2 image (GF-2) was selected in the experiment. The spatial resolution of the image was $1 \mathrm{~m}$ in full color and $4 \mathrm{~m}$ in multi-spectrum. The image acquisition time was 2017-08-04. Due to the dense construction of the color steel buildings in the urban villages of the study area, the single area of the color steel building is relative small. Therefore, firstly, the GF-2 images were subjected to match and fusion processing. secondly, a decision tree model based on multiscale segmentation of the multi-spectral image was used to extract the outlines of the color steel buildings and used the fusion image as a reference and supplemented by manual error correction. Finally, according to the colors and areas, the extracted color steel building information's were classified and coded, to further analyze and process.

Some extracted color steel buildings information based on GF-2 images are shown in Fig. 1 and Fig.2. Blue, highlight white and green spots represent the different types of the colored steel plate buildings on the images. An image of Gansu Agricultural University (GAU), Lanzhou City College (LCU) and the surrounding area (image zoom ratio is 40\%) was shown in Figure 1(a). According to the field surveys, the color steel buildings in the area were mainly distributed in the urban villages around the universities, secondary schools, etc. They mostly were the small color steel buildings for residential and commercial temporary. The outlines of the color steel building were extracted (Figure 1 (b)).

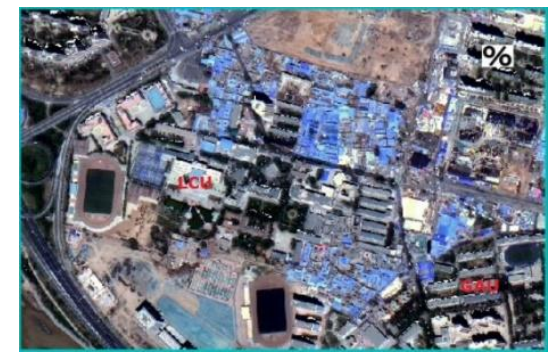

(a) Around GAU/LCU (40\% scaling)

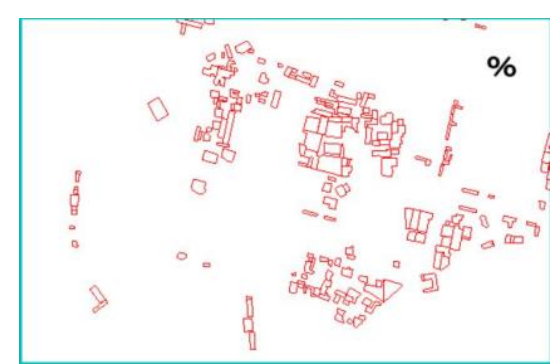

(b) Color steel building extraction results

Figure.1 The small color steel buildings extraction based on the GF-2 images
Figure 2(a) is an image of Gansu Snow Brewery near the site (image scaling is 60\%). The area belongs to the New Technology Development Zone in Anning District, Lanzhou City, and is mostly a large-scale color steel building such as a factory, a company's plant and warehouse. Figure 2 (b) is extracted color steel building information.

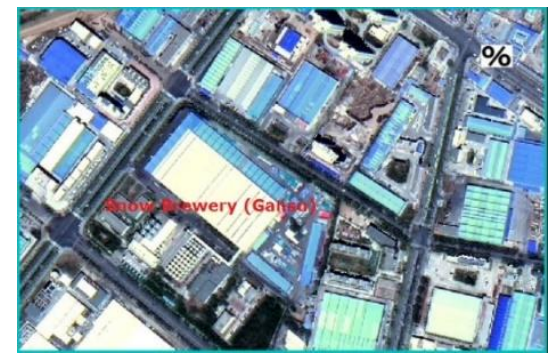

(a) Near Gansu Snow Brewery (60\% scaling)



(b) Color steel building extraction results

Figure. 2 The large color steel buildings extraction based on the GF-2 images

\subsection{Kernel Density Estimation(KDE)}

We analyzed the color steel buildings through the results of the remote sensing interpretation and preliminary, it is found that the color steel buildings had the obvious agglomeration characteristics. Kernel density estimation is one of the most effective methods in point pattern analysis (Elgammal et al., 2002; Xie et al., 2008). Its calculation result can effectively express the feature that the greater the center radiation value is, the closer to the core is (Yu et al., 2015). The method uses a moving window to calculate and export the dot density of each grid cell. For further study, the aggregation and spatial distribution characteristics of the color steel buildings were analyzed this paper by using the geographical statistics analysis method and the nuclear density estimation method.

For kernel density estimation, the better calculation method is defined as:

$$
f(s)=\sum_{i=1}^{n} \frac{1}{h^{2}} k\left(\frac{s-c_{i}}{h}\right)
$$

In the formula, $f(s)$ is the kernel density calculation function at the spatial position $s . h$ is the distance attenuation threshold (bandwidth). $n$ is the number of element points whose distance from the position $s$ is less than or equal to $h$. The $k$ function is the weight function.

Among them, the most commonly used 4 space weight equations are used in the $k$ function.

$$
k\left(\frac{s-c_{i}}{h}\right)=\frac{3}{4}\left(1-\frac{\left(s-c_{i}\right)^{2}}{h^{2}}\right)
$$

According to relevant studies, the choice of a specific weight function has little effect on the density analysis results, and the 
bandwidth $(h)$ value needs to be determined according to the analysis scale and specific issues, directly affecting the results of the density analysis (Davies et al., 201; Chen et al., 2012).

\section{THE ANALYSIS OF SPATIAL DISTRIBUTION AND AGGLOMERATION CHARACTERISTICS}

\subsection{The Analysis of Basic Characteristics of Color Steel Buildings}

The color steel buildings of Anning District of Lanzhou City were extracted based on the decision tree model of the objectoriented multi-scale segmentation, which was used the GF-2 image which was obtained from 2017-08-04. The results were shown in Fig.3.

According to the statistics, there were 4,316 color steel buildings interpreted. According to the actual investigation and image extraction accuracy analysis, the color steel buildings with an area of more than $900 \mathrm{~m}^{2}$ were mainly concentrated in the new technology development zones, and the factories, while warehouses and warehouses of the company were the main products, with a total of 560 buildings. The color steel buildings with an area of less than $900 \mathrm{~m}^{2}$ were scattered in the urban villages and the urban-rural junctions. The temporary buildings were mainly built on residential buildings, with a total of 3756 buildings.

Further statistical analysis was shown in Figure 4. Figure 4(a) showed that the number of small colored steel constructions in the study area was large, accounting for $87 \%$ of all color steel plate constructions. Figure 4(b) showed that the area occupied by the large-scale color steel buildings in the study area was even larger, about $55 \%$.



Figure.3 Extraction results of the color steel buildings in Anning District, Lanzhou City

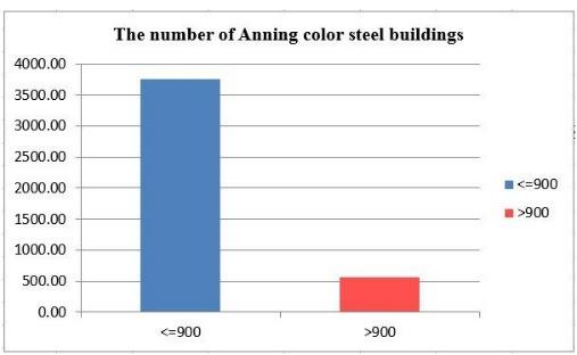

(a) Number differences of color steel buildings

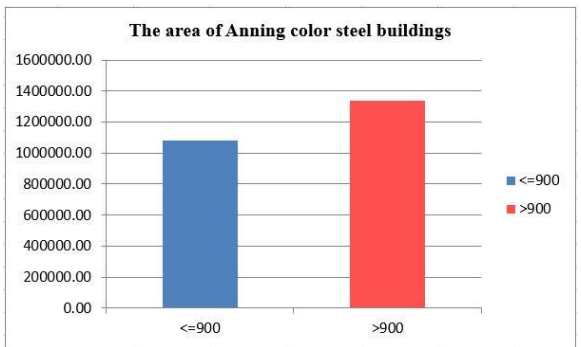

(b) Area differences of color steel buildings

Figure 4 Statistical analysis of the color steel buildings

\subsection{Kernel Density Analysis of Color Steel Building}

Based on the extracted the data of the calm color steel buildings, the kernel density bandwidth $(h)$ adopts an empirical calculation method, according to the spatial distribution and agglomeration characteristics of the color steel buildings (Davies et al., 2018).

$$
h=0.9 \times \min \left(S_{D}, \sqrt{\frac{1}{\ln 2}} \times D_{m}\right) \times n^{-0.2}
$$

In the formula, $n$ is the number of color steel buildings. $D_{m}$ is the median distance from the average center of color steel buildings to each color steel plate building. $S_{D}$ is the standard distance.

In the experiment, firstly, the color steel building data were estimated by the kernel density and the bandwidth was $400 \mathrm{~m}$. The calculated results were shown in Figure 5. The kernel density map effectively demonstrated the spatial distribution and agglomeration characteristics of the color steel buildings in the study area. It is found that the highly concentrated areas of the color steel buildings were mainly located on two types of areas (Figure 5): the first is the surrounding schools which are 
more densely distributed, and the second is a large factory and the company. Because of the concentration of people and logistics in schools and surrounding areas, the rent for fixedconstruction buildings is relatively high. Therefore, the temporary color steel buildings in the surrounding areas (mainly in urban village) are mostly distributed to increase the rent income. There are many factories and warehouses in factories and company-intensive areas. These buildings are mostly largearea fixed color steel plate.

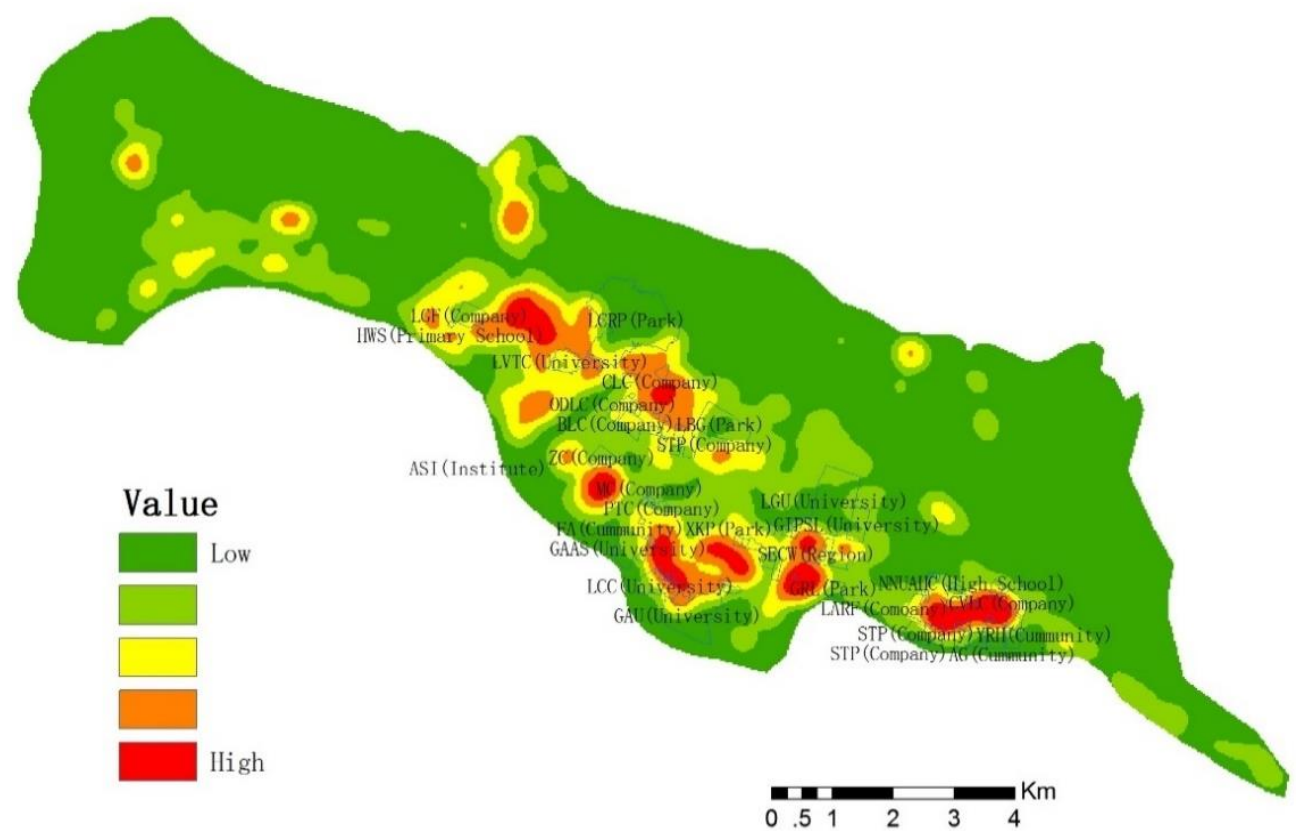

Figure. 5 Distribution of kernel density at the center of color steel buildings

Secondly, based on the above calculations, the kernel density was estimated using the color steel buildings areas as the weight, and the bandwidth was $400 \mathrm{~m}$. The calculation results were shown in Figure 6. This calculation not only took into account the spatial distribution and gathering characteristics of the color steel plate building, but also considered the spatial distribution characteristics of the different types of color steel plate buildings. The areas with the highest kernel density were factories and company clusters from the figure,. Among them, the most obvious areas of agglomeration features were in the streets of Zhongbang Avenue, Lanke Road and Mogao Avenue in Anning New Technology Development Zone of Lanzhou City.



Figure. 6 Distribution of kernel density in color steel building's area 


\section{CONCLUSION}

Through the remote sensing extraction and analysis of the color steel plate construction in the study area, the paper shows that:

(1) Kernel density estimation can effectively analyze the spatial distribution and agglomeration characteristics of the color steel buildings in the study area and can display the aggregation of the different types (through area classification) of color steel buildings to some extent.

(2) In the study area, temporary color steel buildings are densely distributed, mainly in urban villages, urban fringes, and shanty towns, indicating that the research area is still in the transitional stage of urbanization and still has some characteristics of the collective villages and towns.

(3) In the study area, the spatial distribution of large area fixed color steel buildings is relatively concentrated, mainly distributed in the new technology development zone, and the overall area is larger than the temporary color steel plate construction, indicating that the industrialization and commercialization of the research area has reached a certain scale.

In the research process, there are still areas for improvement:

(1) The use of high spatial resolution remote sensing images can effectively identify and extract large-scale color steel building's information, but automated, intelligent extraction algorithms still need to be studied to facilitate the comparative study of multiple time periods in multiple research areas.

(2) The color steel buildings are divided by streets, and the streets have grid features. Therefore, subsequent research using network kernel density and other estimation methods should be able to more effectively analyse the spatial distribution and gathering characteristics of color steel buildings.

(3) Temporary color steel buildings have hidden dangers such as fire and public security. Therefore, it is necessary to conduct correlation analysis on the gathering characteristics for the follow-up study, fire passages, population proportion, and population structure of the temporary color building concentrated areas.

\section{ACKNOWLEDGEMENTS}

The work was financially supported by the National Key R\&D Program of China (2017YFB0504201 and the National Natural Science Foundation of China (No. 41761082).

\section{REFERENCES}

Chen P Y, Zhu X G, 2012a. Regional inequalities in China at different scales. Acta Geographica Sinica, 67(8), pp.1085-1097.

Chen X, 2018a. A spatial and temporal analysis of the socioeconomic factors associated with breast cancer in illinois using geographically weighted generalized linear regression. Journal of Geovisualization and Spatial Analysis, 2(1), 5, pp.216.

Davies T M, Flynn C R, Hazelton M L, 2018a. On the utility of asymptotic bandwidth selectors for spatially adaptive kernel density estimation. Statistics \& Probability Letters, pp.1-7.

Davies T M, Hazelton M L, 2010a. Adaptive kernel estimation of spatial relative risk. Statistics in Medicine, 29(23): 24232437.
Elgammal A, Duraiswami R, Harwood D, et al, 2002a. Background and foreground modeling using nonparametric kernel density estimation for visual surveillance. Proceedings of the IEEE, 90(7), pp.1151-1163.

Feng J, Zhou Y X, 2011a. A review and prospect on urban internal spatial structure research in China. Progress in Geography, 22(3), pp. 304-315.

Herold M, Scepan J, Clarke K C, 2002a. The use of remote sensing and landscape metrics to describe structures and changes in urban land uses. Environment and Planning A, 34(8), pp.1443-1458.

Li R, 2015a. Study of rail transit and urban spatial structure based on urban economics. Urban Transportation \& Construction, 2, pp. 20-22.

Liu Z, He C, Zhang Q, et al, 2012a. Extracting the dynamics of urban expansion in China using DMSP-OLS nighttime light data from 1992 to 2008. Landscape \& Urban Planning, 106(1), pp.62-72.

Pandey B, Joshi P K, Seto K C, 2013a. Monitoring urbanization dynamics in India using DMSP/OLS night time lights and SPOT-VGT data. International Journal of Applied Earth Observation \& Geoinformation, 23(1), pp.49-61.

Shen Y, e Silva J A, Martínez L M, 2014a. Assessing highspeed rail's impacts on land cover change in large urban areas based on spatial mixed logit methods: a case study of Madrid Atocha railway station from 1990 to 2006. Journal of Transport Geography, 41, pp.184-196.

Shi K, Yu B, Huang Y, et al, 2014a. Evaluating the ability of NPP-VIIRS nighttime light data to estimate the gross domestic product and the electric power consumption of China at multiple scales: A comparison with DMSP-OLS data. Remote Sensing, 6(2), pp. 1705-1724.

Woo M, Guldmann J M, 2011a. Impacts of urban containment policies on the spatial structure of US metropolitan areas. Urban Studies, 48(16), pp. 3511-3536.

Xie Z, Yan J, 2008a. Kernel density estimation of traffic accidents in a network space. Computers, environment and urban systems, 32(5), pp.396-406.

Yin M, Bertolini L, Duan J, 2015a. The effects of the highspeed railway on urban development: international experience and potential implications for China. Progress in Planning, 98, pp.1-52.

Yu W, AI T, Liu P, et al, 2015a. Network kernel density estimation for the analysis of facility POI hot spots. Acta Geodaetica et Cartographica Sinica, 44(12), pp. 1378-1383.

Zhang X H, Zhang X, Zhong E S, et al, 2013a. Study on the spatial structure and driving force of urban areas in Beijing based on building space structure. Geographical research, 32(11), pp. 2055-2065. 